\title{
RETINAL THICKNESS ESTIMATION FROM SD-OCT MACULAR SCANS
}

\author{
N. Hammes ${ }^{1}$, L. Racette ${ }^{2}$, B.C. Samuels ${ }^{3}$, and G. Tsechpenakis ${ }^{1}$ \\ ${ }^{1}$ Computer and Information Science Department, Indiana University-Purdue University Indianapolis, USA \\ ${ }^{2}$ Department of Ophthalmology, Indiana University School of Medicine, USA \\ ${ }^{3}$ Department of Ophthalmology, University of Alabama at Birmingham School of Medicine, USA
}

\begin{abstract}
Glaucoma, a leading cause of blindness worldwide, can be detected using retinal thicknesses from spectral-domain optical coherence tomography (SD-OCT) scans of the macula. We calculate the desired thickness maps as the distance between the inner-limiting membrane (ILM) and retinal pigmented epithelium (RPE) of the retina. To delineate these two layers, we use a set of two deformable open surfaces that are driven by intensity contrast, while preserving their shape and topology properties, i.e. local surface smoothness and inter-surface distance smoothness. To evaluate our method, qualified graders manually segmented 30 random sections from 20 OCT image stacks, in triplicate; we make comparisons with obtained ground-truth and the clinically tested Heidelberg Spectralis segmentation. We show the superiority of our method with respect to accuracy and average execution time ( $\sim 7 \mathrm{secs})$, validating it as a clinical tool.
\end{abstract}

Index Terms - Retinal thickness, early detection of glaucoma, interacting deformable surfaces

\section{INTRODUCTION}

Glaucoma is one of the leading causes of significant vision loss and blindness throughout the world $[6,13]$. The disease is characteristically defined as a chronic optic neuropathy that results in the loss of retinal ganglion cells and their axons (i.e. retinal nerve fiber layer; RNFL), with increased intraocular pressure being the primary risk factor. It is the cumulative loss of these retinal ganglion cells that leads to permanent visual field defects and eventual blindness. Thus, the goal of clinicians is to detect glaucoma as early as possible in the disease process in order to preserve visual function.

New advances in technology have resulted in the development of quicker, high-definition spectral-domain optical coherence tomography (SD-OCT) imaging with retinal image resolution of $3.9 \mu \mathrm{m} \mathrm{[10].} \mathrm{Glaucoma} \mathrm{analysis} \mathrm{software}$ has been developed to examine for glaucomatous retinal defects by identifying loss of macular retinal thickness and asymmetry between the superior and inferior hemifields [1]. Total retinal thickness is calculated as the distance between the inner-limiting membrane (ILM), the interface between the dark vitreous space and the bright RNFL, and the highly reflective retinal pigmented epithelium (RPE), the last clear boundary between retina and the choroid vessels (Fig. 1(a)).



(a)



(b)
Fig. 1: Retinal images taken from within an SD-OCT image stack. Note Cartesian axes for future reference. (a) Purple lines denote the two layers of interest: inner-limiting membrane (ILM) above, retinal pigmented epithelium (RPE) below. Red arrows indicate potential challenges for ILM estimation: (i) vitreous artifact at left presents an area of continuous contrast similar to the ILM; (ii) topological dip at the foveola is often accompanied by a reduction in absolute contrast, making a concrete measure of contrast impractical. Green arrows indicate potential issues for capturing RPE: both the choroid (left) and inner/outer photoreceptor segment junction (right) provide areas of contrast similar to that of RPE. (b) Colored grids demonstrate the result for a 3-dimensional segmentation of the two layers.

Since the advent of SD-OCT, various automatic segmentation algorithms have been employed to determine the layers of the human retina and the corresponding thicknesses between them. A recent review article [2] categorizes these methods into appearance classification, deformable models, global optimization using graph cuts, and modelfitting/registration methods. Supervised classification methods, specifically support vector machines, are effective in obtaining desired boundaries [15]. For analysis of image stacks (3D), however, the computational complexity is beyond the maximum allowable segmentation time in clinical settings; additionally, the non-uniform noise throughout the volume is a challenge for sufficient sampling during supervised learning. Model-fitting and registration methods use a predetermined shape to fit to the data. These methods are usually computationally efficient, but lack robustness when dealing with shape formations beyond the expected (trained) variations [4]. Lastly global optimization methods mainly arise from the utilization of the max-flow min-cut algorithm commonly paired with graph models $[7,5]$, they can be computationally efficient and robust to local observation variations, but they do not incorporate shape and domain-specific topology information.

The realm of deformable models can be divided into two classes: the implicit or geometric models, and the parametric models. Currently, only a few deformable model-based approaches have been used to segment various aspects of OCT

This is the author's manuscript of the article published in final edited form as: 
images, all belonging to the parametric family. In 2005, Cabrera et al. [3] illustrated the utility of parametric deformable models through demonstration of the accurate segmentation of the fluid-filled regions common in the OCT's of patients with age-related macular degeneration. That same year, Mujat et al. [12] investigated a deformable model using splines for retinal layer segmentation, but limited their analysis of SD-OCT stacks to sequential 2D analysis of the images, with total segmentation time for a stack being $\sim 62$ seconds. In 2009, Mishra et al. [11] developed an active contour-based algorithm to segment multiple layers in time-domain OCT, a precursor of the significantly higher resolution SD-OCT technology. Reported segmentation time was considered 'highly efficient', at 5 seconds per 2-dimensional image, also technically known as a B-scan.

The parametric deformable models can be subdivided into region-based and edge-based methods. The most recent example of region-based deformable model segmentation is that of Yazdanpanah et al. [16], where standalone murine SDOCT B-scans were segmented into 6 retinal layers. As reported in [16], this model can be corrupted by noise and suffers from areas of low contrast, while the segmentation time was not provided. Such model-based approaches, while effective with respect to a single image of an OCT stack, ignore useful spatial information that could be harnessed if an integrated 3D volume analysis was performed.

This article introduces a fast hybrid method designed to evolve two 2D spline-defined surfaces to the ILM and RPE for the purpose of retinal thickness calculation. Our framework exploits intrinsic smoothness constraints within and between the sheet-like models, and is driven by contrast in a 'traditional' energy minimization fashion. We aim at using our method as a clinical tool, replacing the the state-of-theart in clinically tested software, namely the native Heidelberg Spectralis segmentation [10].

\section{METHOD}

Our model consists of two open surfaces $S_{\text {ILM }}$ and $S_{\mathrm{RPE}}$ that evolve in the volume domain $\Omega$ to capture the two retinal layers, the inner-limiting membrane (ILM) and the retinal pigmented epithelium (RPE), respectively. The vertical distance between $S_{\mathrm{ILM}}$ and $S_{\mathrm{RPE}}$ (Fig. 1: distance in Z), the retinal thickness, is the spatial value map of interest for this clinical application. The surfaces are initially considered as 'flat sheets', i.e., each one is parallel to an XY plane, $S_{\text {ILM }}$ moves downwards and $S_{\mathrm{RPE}}$ moves upwards along the Zaxis, and their shapes deform locally according to data contrast, lattice-based deformations, and their mutual distance (Fig. 1(b): colored grids). Note that a typical SD-OCT volume is $768 \times 61 \times 496(\mathrm{XYZ})$ voxels.

\subsection{Topolgy}

The two surfaces, $S_{\mathrm{ILM}}$ and $S_{\mathrm{RPE}}$, are interconnected through their signed distance transforms (level set-like implicit shape

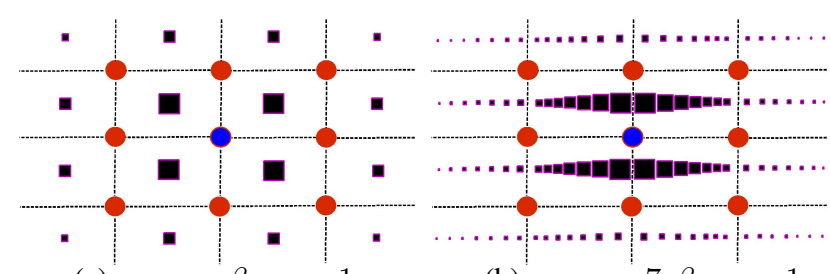

(a) $\alpha_{\mathrm{ILM}}=\beta_{\mathrm{ILM}}=1$

(b) $\alpha_{\mathrm{RPE}}=7, \beta_{\mathrm{ILM}}=1$

Fig. 2: Weight maps used to impart deformations to control points of a lattice by surrounding voxels. The deformation of the blue control point at the center is derived by weighting the deformation values of the surrounding voxels (black squares), as shown proportional to size (voxel distances are normalized). (a) For the surface $S_{\text {ILM }}$ evolving towards the ILM, each deformation is more 'flexible' with 16 voxels influencing each control point. (b) Lattice spacing for the surface $S_{\mathrm{RPE}}$ evolving towards the RPE allows for more voxels to contribute to each control point's deformation. This adds smoothness to the model at the expense of flexibility. We chose the specific spacing parameters, considering the data ambiguities around RPE, to make our solution more robust to noise (also see Fig. 1).

representations) $\Phi_{\mathrm{ILM}}$ and $\Phi_{\mathrm{RPE}}$, respectively. These transforms are considered to have the following conditional breakdown, $\forall \mathbf{x} \in \Omega$ :

$$
\begin{aligned}
& \Phi_{\mathrm{ILM}}(\mathbf{x})=\left\{\begin{aligned}
0, & \mathbf{x} \in S_{\mathrm{ILM}} \\
+\min _{\mathbf{x}_{\mathrm{ILM}} \in S_{\mathrm{ILM}}}\left\|\mathbf{x}-\mathbf{x}_{\mathrm{ILM}}\right\|, & \mathbf{x} \in S_{\mathrm{ILM}}^{+} \\
-\min _{\mathbf{x}_{\mathrm{ILM}} \in S_{\mathrm{ILM}}}\left\|\mathbf{x}-\mathbf{x}_{\mathrm{ILM}}\right\|, & \mathbf{x} \in S_{\mathrm{ILM}}^{-}
\end{aligned}\right. \\
& \Phi_{\mathrm{RPE}}(\mathbf{x})=\left\{\begin{aligned}
0, & \mathbf{x} \in S_{\mathrm{RPE}} \\
+\min _{\mathbf{x}_{\mathrm{RPE}} \in S_{\mathrm{RPE}}}\left\|\mathbf{x}-\mathbf{x}_{\mathrm{RPE}}\right\|, & \mathbf{x} \in S_{\mathrm{RPE}}^{+} \\
-\min _{\mathbf{x}_{\mathrm{RPE}} \in S_{\mathrm{RPE}}}\left\|\mathbf{x}-\mathbf{x}_{\mathrm{RPE}}\right\|, & \mathbf{x} \in S_{\mathrm{RPE}}^{-}
\end{aligned}\right.
\end{aligned}
$$

where $\left\{S_{\mathrm{ILM}}^{+}, S_{\mathrm{ILM}}^{-}\right\}$are the volumes above and below $S_{\mathrm{ILM}}$ respectively, and $\left\{S_{\mathrm{RPE}}^{+}, S_{\mathrm{RPE}}^{-}\right\}$are the volumes below and above $S_{\mathrm{RPE}}$ respectively, along the Z-axis. Note that the positive sign of the distances corresponds to the volume parts already passed through during the downward $\left(S_{\text {ILM }}\right)$ /upward ( $S_{\mathrm{RPE}}$ ) evolution, i.e., each surface moves towards negative distance values. Thus, we encode the relative topology between the two surfaces as,

$$
\Phi_{t}(\mathbf{x})=\Phi_{\mathrm{ILM}}(\mathbf{x})+\Phi_{\mathrm{RPE}}(\mathbf{x}), \quad \forall \mathbf{x} \in \Omega
$$

Intuitively, $\Phi_{t}$ encodes the local (with respect to XY) distance between $S_{\mathrm{ILM}}$ and $S_{\mathrm{RPE}}$ along the Z-axis; positive values of $\Phi_{t}$ indicate that the two surfaces have crossed each other locally, which violates the layer topology constraint.

\subsection{Local deformations}

The inner-limiting membrane (ILM) and the retinal pigmented epithelium (RPE) surfaces, $S_{\mathrm{ILM}}$ and $S_{\mathrm{RPE}}$, deform locally using free-form deformations (FFD) $[14,8]$. The two lattices used for such deformations are parametrized by $\left\{\alpha_{\mathrm{ILM}}, \beta_{\mathrm{ILM}}\right\}$ and $\left\{\alpha_{\mathrm{RPE}}, \beta_{\mathrm{RPE}}\right\}$, where the $\alpha$ and $\beta$-values are integers determining spacing between control points along $\mathrm{X}$ and $\mathrm{Y}$ directions respectively. Thus, a single control-point deformation is calculated using a weighted map derived from cubic B-spline functions (Fig. 2). This map gives more weight to the voxels in close proximity to 
the control point, and varies with the chosen XY-spacing of the lattice. In our application, we constrain the local deformations of $S_{\mathrm{RPE}}$, due to the increased noise around RPE in the OCT volumes. In our experiments we used $\alpha_{\mathrm{RPE}}=7$ and $\beta_{\mathrm{RPE}}=1$. On the other hand, we allow for larger local deformations of $S_{\mathrm{ILM}}$, given that there is sufficient contrast information around ILM. Additionally, ILM displays local changes in topology due to the presence of blood vessels just under the surface; therefore, a spacing of $\alpha_{\mathrm{ILM}}=\beta_{\mathrm{ILM}}=1$ allows flexibility and more finely grained deformations to capture such local changes.

\subsection{Objective function and evolution}

The two surfaces $S_{\mathrm{ILM}}$ and $S_{\mathrm{RPE}}$ evolve simultaneously along the Z-axis towards the desired ILM and RPE boundaries, driven by intensity contrast in the OCT volume, local shape smoothness dictated by the free-form deformations within their lattices, and the relative topology (Z-distance) between them. We formulate the objective energy as,

$$
\begin{aligned}
E_{\text {total }}=E_{\text {shape }}\left(\Phi_{\mathrm{ILM}}, \Phi_{\mathrm{RPE}}\right)+ & E_{\text {top }}\left(\Phi_{\mathrm{ILM}}, \Phi_{\mathrm{RPE}}\right)+ \\
& +E_{\text {data }}\left(\Phi_{\mathrm{ILM}}, \Phi_{\mathrm{RPE}}\right),
\end{aligned}
$$

where the shape and topology energy terms, $E_{\text {shape }}$ and $E_{\text {top }}$, are considered as the internal energy of a deformable model consisting of two evolving surfaces, while the data energy $E_{\text {data }}$ is the external energy, introducing the data intensity contrast that drives the evolution.

Shape energy. Following a similar formulation as in [8], let $d_{\mathrm{ILM}}$ and $d_{\mathrm{RPE}}$ be the deformation (distance) parameters of the lattices defined for ILM and RPE respectively. Note that for efficiency we decouple XY deformations from the evolution along the Z-direction in the volume, by defining $2 \mathrm{D}$ instead of 3D lattices. We formulate the shape-based energy term as,

$$
\begin{aligned}
E_{\text {shape }}\left(\Phi_{\mathrm{ILM}}, \Phi_{\mathrm{RPE}}\right) & =\frac{1}{\mathcal{V}\left(\partial S_{\mathrm{ILM}}\right)} \int_{\partial S_{\mathrm{ILM}}} \Phi_{\mathrm{ILM}}(\mathbf{x}) d \mathbf{x}+ \\
& +\frac{1}{\mathcal{V}\left(\partial S_{\mathrm{RPE}}\right)} \int_{\partial S_{\mathrm{RPE}}} \Phi_{\mathrm{RPE}}(\mathbf{x}) d \mathbf{x},
\end{aligned}
$$

where $\partial S_{\text {ILM }}, \partial S_{\mathrm{RPE}}$ denote narrow bands, with respect to $\mathrm{Z}$, around the evolving surfaces, and $\mathcal{V}\left(\partial S_{\mathrm{ILM}}\right), \mathcal{V}\left(\partial S_{\mathrm{RPE}}\right)$ are their corresponding volumes. Based on the definitions in eqs. (1) and (2), minimization of this energy corresponds to moving $S_{\text {ILM }}$ downwards and $S_{\mathrm{RPE}}$ upwards along the Zdirection. For notation simplicity, $\mathbf{x}$ incorporates the FFD parameters $d_{\mathrm{ILM}}$ and $d_{\mathrm{RPE}}$ that inherently preserve continuity.

Topology energy. We define this energy term as a functional of hyperbolic nature, where both the absolute value and the sign of $\Phi_{t}(\mathbf{x}), \forall \mathbf{x} \in \Omega$, contribute. Specifically, small values of $\left|\Phi_{t}\right|$ correspond to close proximity of the two surfaces. Also, according to the definitions in eqs. (1)-(3), positive values of $\Phi_{t}$ indicate that the two surfaces cross each other or even that RPE is above ILM with respect to the Z-axis, which violates the inherent topology constraints of the two retina layers. Therefore, minimization of the topology energy should force the two surfaces closer along $\mathrm{Z}$, while preserving negative values of $\Phi_{t}$. We formulate this energy as,

$$
\begin{gathered}
E_{\mathrm{top}}\left(\Phi_{\mathrm{ILM}}, \Phi_{\mathrm{RPE}}\right)= \\
E_{\mathrm{top}}\left(\Phi_{t}\right)=\int_{\Omega} \Phi_{t}^{2}(\mathbf{x}) \cdot \mathcal{H}\left[\Phi_{t}(\mathbf{x})\right] d \mathbf{x}, \\
\mathcal{H}\left[\Phi_{t}(\mathbf{x})\right]=\left\{\begin{array}{cc}
1, & \Phi_{t}(\mathbf{x}) \leq 0 \\
h \gg 1, & \Phi_{t}(\mathbf{x})>0
\end{array}, \forall x \in \Omega\right.
\end{gathered}
$$

where $h$ is a large positive number that controls the energy increase for positive values of $\Phi_{t}: h \rightarrow \infty \Rightarrow E_{\text {top }}\left(\Phi_{t} \rightarrow\right.$ $\left.0^{+}\right) \rightarrow \infty$. A reasonable choice is $h \geq 100$.

Data energy. A first observation in the OCT volumes is that the first instance of intensity contrast, traversing from top to bottom along $\mathrm{Z}$, is the ILM, while the last instance of high contrast is the RPE (Fig. 1). Let $g=\left|\nabla_{z} I\right|$ denote the magnitude of the intensity gradient of the data volume $I$ along the $\mathrm{Z}$-axis (note we are interested in capturing high vertical contrast instances). We quantify instances of high contrast as a 'reliability' map,

$$
G(\mathbf{x})=\left\{\begin{array}{rl}
\exp \left\{-\left(g(\mathbf{x})-\left.\bar{g}\right|_{\mathbf{x}}\right)^{2}\right\}, & g(\mathbf{x})<\left.\bar{g}\right|_{\mathbf{x}} \\
g(\mathbf{x}), & g(\mathbf{x}) \geq\left.\bar{g}\right|_{\mathbf{x}}
\end{array},\right.
$$

where $\left.\bar{g}\right|_{\mathbf{x}}$ denotes the average value of gradient $g$ in $\mathbf{x}$ 's vicinity. Thus, we formulate the data energy term as,

$$
\begin{array}{r}
E_{\text {data }}\left(\Phi_{\text {ILM }}, \Phi_{\mathrm{RPE}}\right)=\frac{1}{\exp \left\{\lambda G^{2}(\mathbf{x})\right\}}, \\
\forall \mathbf{x} \in \Omega: \Phi_{\mathrm{ILM}}(\mathbf{x})=0 \text { or } \Phi_{\mathrm{RPE}}(\mathbf{x})=0,
\end{array}
$$

where $\lambda$ controls the decrease slope as $G^{2}$ increases; in all our experiments we chose $\lambda=10$.

Model evolution. As we mention above, there are two factors contributing to the model evolution: the local deformations $\mathbf{d}=\left\{d_{\mathrm{ILM}}, d_{\mathrm{RPE}}\right\}$ of the ILM and RPE lattices, and movement along the Z-axis of the OCT volume. Therefore, the minimization of the energy in eq. (4) that drives the evolution of the two surfaces is expressed as,

$$
\frac{\partial E_{\text {total }}}{\partial \mathbf{d} \partial z}=\frac{\partial E_{\text {shape }}}{\partial \mathbf{d} \partial z}+\frac{\partial E_{\text {top }}}{\partial \mathbf{d} \partial z}+\frac{\partial E_{\text {data }}}{\partial \mathbf{d} \partial z}
$$

We solve the minimization problem using a greedy algorithm locally, i.e., iteratively in narrow (along Z) sub-volumes, defined by a fixed depth value $\delta z$ around the evolving surfaces: (a) first we initialize the deformation parameters for both surfaces as $\mathbf{d}=0$; (b) we compute the distance and topology functions in eqs. (1)-(3), and the contrast reliability map in eq. (8); (c) locally, we compute $\left\langle\hat{\Phi}_{\mathrm{ILM}}, \hat{\Phi}_{\mathrm{RPE}}\right\rangle_{z}=\arg \min _{z \in \delta z} E_{\text {total }}$; (d) at the new depth locations we compute the lattice configurations and the resulting distance maps as, $\left\langle\hat{\Phi}_{\mathrm{ILM}}, \hat{\Phi}_{\mathrm{RPE}}\right\rangle_{\mathbf{d}}=\arg \min _{\mathbf{d}} E_{\text {total }}$; (e) at the new Z-locations with the given deformation parameters, we repeat (b)-(d) until convergence.

\section{RESULTS}

Ten healthy study participants were given bilateral macular SD-OCT scans using the Heidelberg Spectralis SD-OCT, 


\begin{tabular}{lll|l|l} 
Grader & Method & $\begin{array}{l}\text { ILM } \\
\text { (mean, dev.) }\end{array}$ & $\begin{array}{l}\text { RPE } \\
\text { (mean, dev.) }\end{array}$ & $\begin{array}{l}\text { Thickness } \\
\text { (Mean, dev.) }\end{array}$ \\
\hline \multirow{2}{*}{ Both } & Ours & $(3.33,2.64)$ & $(4.23,2.77)$ & $(6.61,3.90)$ \\
& Heidelberg & $(2.74,2.36)$ & $(6.71,2.26)$ & $(8.57,3.63)$ \\
\hline \multirow{2}{*}{ Gr. 1 } & Ours & $(2.90,2.58)$ & $(3.59,2.63)$ & $(5.30,3.71)$ \\
& Heidelberg & $(2.48,2.28)$ & $(5.94,2.32)$ & $(6.98,3.72)$ \\
\hline \multirow{2}{*}{ Gr. 2 } & Ours & $(4.07,2.94)$ & $\begin{array}{l}(5.04,3.20) \\
(7.49,2.78)\end{array}$ & $\begin{array}{l}(8.23,4.36) \\
(10.23,4.10)\end{array}$
\end{tabular}

Table 1: Mean errors and standard deviations of our method and the Heidelberg Spectralis native software, as compared to manual delineations of two graders. All values are in $\mu \mathrm{m}$, and for the given application it is $3.8717 \mu \mathrm{m}=1$ voxel.

resulting in 20 full image stacks of $768 \times 61 \times 496$ voxels. Spectralis segmentation results for the ILM and the RPE were extracted from native Heidelberg Eye Explorer (HEYEX) .vol software files using the ImageJ plugin Open Heyex Raw [9]. Our segmentation results were obtained using an Intel i7 $2.8 \mathrm{GHz}$ laptop, and the average segmentation time for a given image stack was 7 seconds. It should be noted that although the optic nerve head $(\mathrm{ONH})$ is present at least partially in all macular images taken by the Spectralis, such areas were removed from statistical consideration for this study. Thickness measurements around the optic nerve head are not used in the clinical testing for which this method is designed; we did not evaluate the suitability of our method for estimating thickness near the $\mathrm{ONH}$.

Two qualified graders manually traced the RPE and ILM in 30 images chosen randomly from the set of 20 macular image stacks. Segmentation was performed in triplicate for each of the 30 images to account for intra-grader variability. To demonstrate segmentation efficacy, we define as segmentation error the voxel difference from the ground-truth along the Z-axis. We compare our method with (Table 1): (i) the native Heidelberg Spectralis software, the current state-of-theart, (ii) the first and second grader delineations, and (iii) the average delineations of both graders. The first 2 rows of Table 1 summarize the main points of the results. For the ILM, the Spectralis software mean error is slightly better compared to ours. The RPE results show much less error for our method compared to the Spectralis software. This results in an overall better fit of our method over the Spectralis when considering the retinal thickness calculation, as shown in the last two columns of the table.

In Fig. 3 we illustrate a qualitative evaluation of our method, compared to the ground-truth and the Spectralis software. In panel (a) we show a section of our converged model (in magenta color) in a highly degraded scan from a glaucoma patient. Even in instances of relatively low contrast, usually resulting from layer thinning, as observed by lower light reflection, such as the left-most part of RPE (lower layer), we are able to capture the correct locations. In panel (b) we illustrate a B-scan to visually showcase the voxel-wise accuracy of our method in the OCT stack; we focus on the region highlighted in the yellow box. The two experts' manual delineations (three for each grader) inside this region are shown
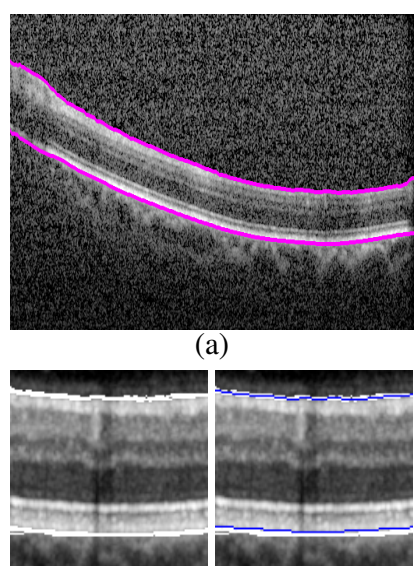

(a)

(c)

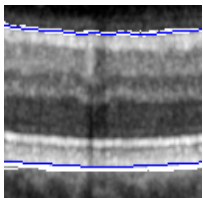

(d)

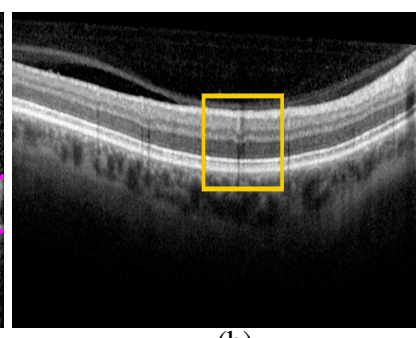

(b)

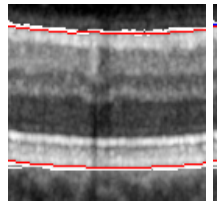

(e)

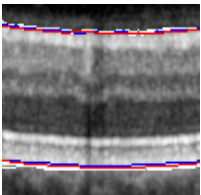

(f)
Fig. 3: Qualitative evaluation of our method. (a) A cross-section of our converged model (magenta lines) in a degraded OCT B-scan. (b) A common-case B-scan. (c) Six manual delineations from two experts, in white, in a magnified instance of the region in the yellow box in (b). (d) The result of Heidelberg Spectralis native software in blue, superimposed on the experts traces. (e) Cross-section of our converged model, in red, superimposed on the experts' traces. (f) Results of our method (in red) and Spectralis (in blue), both superimposed on the same region to visually identify deviations from ground-truth (in white).

with white lines in panel (c); in this case there is significant overlap/agreement between the manual traces. In panels (d) and (e) we show the Spectralis (in blue) and our method's (in red) results, respectively, superimposed on the graders' traces. In (f) we summarize the comparison from (d), (e).

We should note that our method could become more accurate and robust to noise at the expense of computational efficiency, by using machine learning to drive the model. However our goal here is to provide the best possible trade-off between accuracy and efficiency.

\section{CONCLUSIONS}

We presented a model-based method for the segmentation of the inner and outer layers of the retina, namely the innerlimiting membrane (ILM) and retinal pigmented epithelium (RPE), from SD-OCT image stacks. Contrary to previous studies using 2D deformable models, here we exploit the intensity continuity across the successive B-scans and obtain directly $3 \mathrm{D}$ results (surfaces for the layers of interest), while preserving computational times low enough for clinical settings. We calculate the vertical distance between the two surfaces to provide the retinal thickness used for early detection of glaucoma. The eventual goal of our clinical study is the longitudinal evaluation of RNFL thickness changes in a larger subset of healthy and glaucoma patients. The method presented here covers significant ground in this direction. Future work also includes how our method can be adapted in cases beyond healthy and glaucoma patients, such as macular holes and dry age-related macular degeneration. 


\section{REFERENCES}

[1] Asrani S., Rosdahl J.A., Allingham R.R., 'Novel software strategy for glaucoma diagnosis: asymmetry analysis of retinal thickness,' Arch. Ophthalmol, 129(9):1205-1211, 2011.

[2] Cabrera DeBuc D., A Review of Algorithms for Segmentation of Retinal Image Data Using Optical Coherence Tomography, Image Segmentation, Ho P.-G. (Ed.), InTech, 2011, pp.15-54.

[3] Cabrera Fernández D., Salinas H.M., Puliafito C.A., 'Automated detection of retinal layer structures on optical coherence tomography images,' Opt. Express, 13(25):10200-10216, 2005.

[4] Chen M., Lang A., Sotirchos E., Ying H.S., Calabresi P.A., Prince J.L., Carass, A., 'Deformable registration of macular oct using a-mode scan similarity,' Int'l Symp. Biomed. Imaging (ISBI), pp.476-479, 2013.

[5] Chiu S.J., Li C.T., Nicholas P., Toth C.A., Izatt J.A., Farsiu S., 'Automatic segmentation of seven retinal layers in SDOCT images congruent with expert manual segmentation,' Opt. Express, 18:19413-19428, 2010.

[6] Friedman D.S., Wolfs R.C., O’Colmain B.J., Klein B.E., Taylor H.R., West S., Leske M.C, Mitchell P., Congdon N., Kempen J., 'Prevalence of open-angle glaucoma among adults in the United States,' Arch. Ophthalmol., 122: 532-538, 2004.

[7] Garvin M.K., Abramoff M.D., Wu X., Russell S.R., Burns T.L., Sonka M., 'Automated 3-D intraretinal layer segmentation of macular spectral-domain optical coherence tomography images,' IEEE Trans. Med. Imaging 28(9):1436-1447, 2009.

[8] Huang X., Metaxas D., 'Metamorphs: Deformable shape and appearance models,' IEEE Trans. Pattern Analysis and Machine Intelligence, 30(8):1444-1459, 2008.

[9] Knott E.J., Sheets K.G., Zhou Y., Gordon W.C., Bazan N.G., 'Spatial correlation of mouse photoreceptor-RPE thickness between SD-OCT and histology,' Experimental Eye Research, 92(2):155-160, 2011.

[10] Leite M.T., Rao H.L., Zangwill L.M., Weinreb R.N., Medeiros F.A., 'Comparison of the diagnostic accuracies of the Spectralis, Cirrus, and RTVue optical coherence tomography devices in glaucoma,' Ophthalmology, 118(7):1334-1339, 2011.

[11] Mishra A., Wong A., Bizheva K., Clausi D.A., 'Intra-retinal layer segmentation in optical coherence tomography images,' Opt. Express, 17:23719-23728, 2009.

[12] Mujat M., Chan R., Cense B., Park B., Joo C., Akkin T., Chen T., de Boer J., 'Retinal nerve fiber layer thickness map determined from optical coherence tomography images,' Opt. Express, 13(23):9480-9491, 2005.

[13] Quigley H.A., Broman A.T., 'The number of people with glaucoma worldwide in 2010 and 2020,' Br J Ophthalmol, 90:262267, 2006.

[14] Sederberg T.W., Parry S.R., 'Free-form deformation of solid geometric models,' ACM Conf. on Computer Graphics and Interactive Techniques (SIGGRAPH), pp. 151-160, 1986.

[15] Srinivasan P., Heflin S., Izatt J., Arshavsky V., Farsiu S., 'Automatic segmentation of up to ten layer boundaries in SD-OCT images of the mouse retina with and without missing layers due to pathology,' Biomed. Opt. Express, 5:348-365, 2014.

[16] Yazdanpanah A., Hamarneh G., Smith B., Sarunic M., 'Intraretinal layer segmentation in optical coherence tomography using an active contour approach,' Med Image Computing and Computer Assisted Intervention (MICCAI), 12:649-656, 2009. 